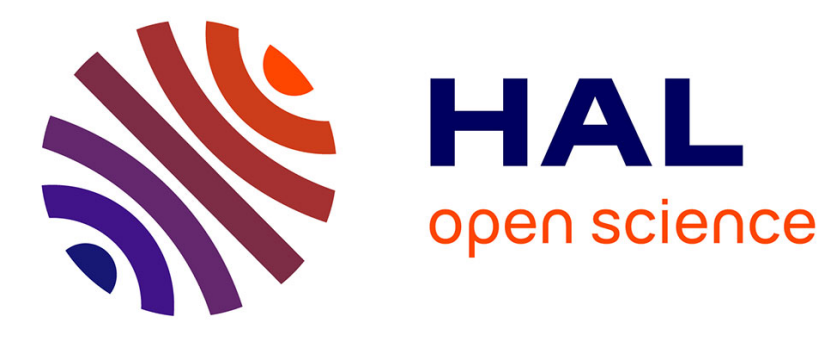

\title{
Design of automotive X-by-Wire systems
}

Cédric Wilwert, Nicolas Navet, Ye-Qiong Song, Françoise Simonot-Lion

\section{To cite this version:}

Cédric Wilwert, Nicolas Navet, Ye-Qiong Song, Françoise Simonot-Lion. Design of automotive X-byWire systems. Richard Zurawski. The Industrial Communication Technology Handbook, CRC Press, 2005, 0849330777. inria-00000562

\section{HAL Id: inria-00000562 https://hal.inria.fr/inria-00000562}

Submitted on 27 Aug 2007

HAL is a multi-disciplinary open access archive for the deposit and dissemination of scientific research documents, whether they are published or not. The documents may come from teaching and research institutions in France or abroad, or from public or private research centers.
L'archive ouverte pluridisciplinaire HAL, est destinée au dépôt et à la diffusion de documents scientifiques de niveau recherche, publiés ou non, émanant des établissements d'enseignement et de recherche français ou étrangers, des laboratoires publics ou privés. 


\section{Design of automotive $\mathrm{X}$-by-Wire systems}

Cédric Wilwert

PSA Peugeot - Citroën

92000 La Garenne Colombe - France

Fax: +33 383581701

Phone: +33 383581717

Cedric.Wilwert@mpsa.com

Nicolas Navet

LORIA UMR 7503 - INRIA

Campus Scientifique - BP 239 - 54506 VANDOEUVRE-lès-NANCY CEDEX

Fax: +33 383581701

Phone : +33383581761

nnavet@,loria.fr

Ye Qiong Song

LORIA UMR 7503 - Université Henri Poincaré Nancy I

Campus Scientifique - BP 239 - 54506 VANDOEUVRE-lès-NANCY CEDEX

Fax: +33383581701

Phone : +33 383581764

song@,loria.fr

Françoise Simonot-Lion

LORIA UMR 7503 - Institut National Polytechnique de Lorraine

Campus Scientifique - BP 239 - 54506 VANDOEUVRE-lès-NANCY CEDEX

Fax: +33 383278319

Phone : +33 383581762

simonot@,loria.fr

\section{CONTENTS}

Design of automotive X-by-Wire systems

Abstract :

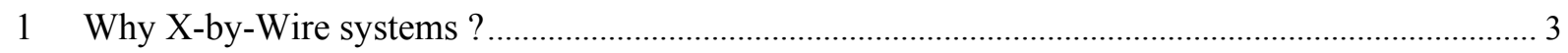

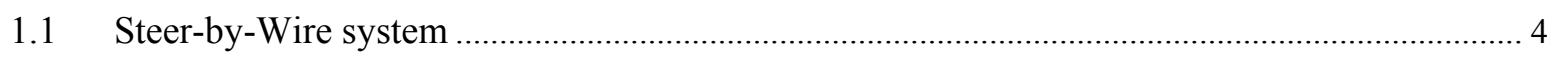

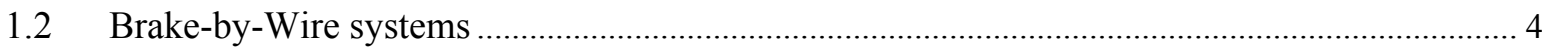

2 Problem, context and constraints for the design of X-by-Wire systems ............................................ 5

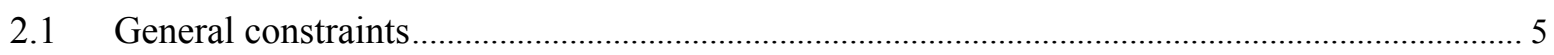

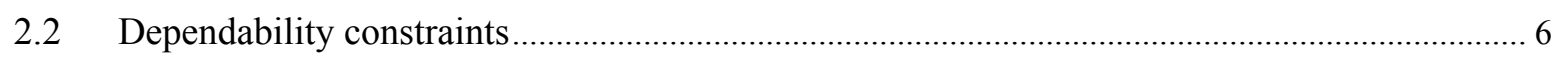

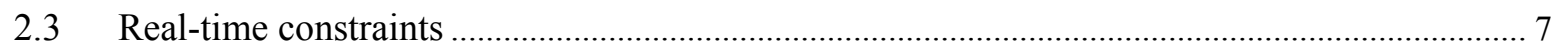

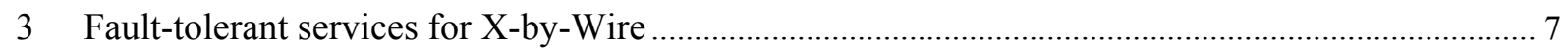




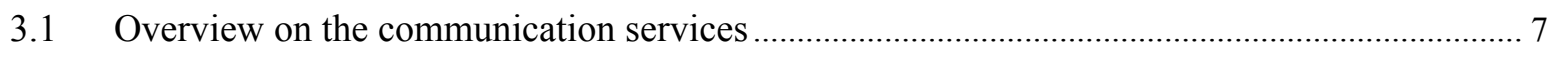

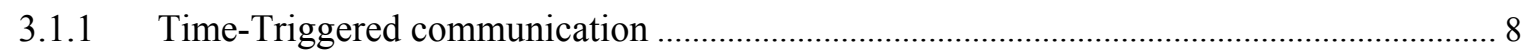

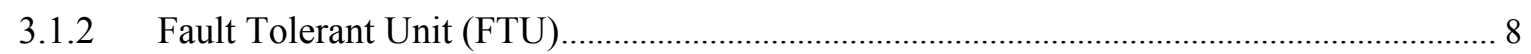

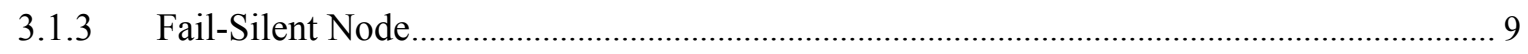

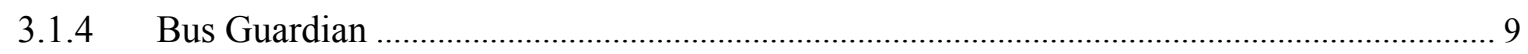

3.2 Main time-triggered protocols for automotive industry ........................................................ 10

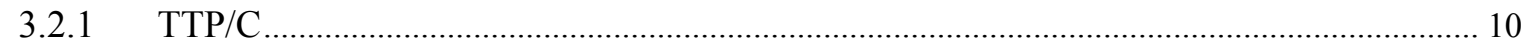

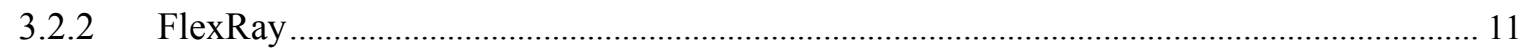

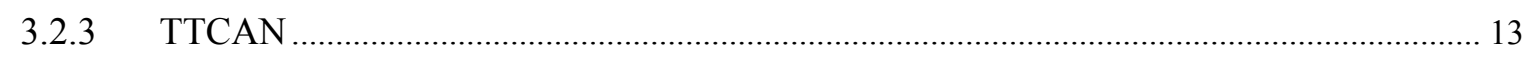

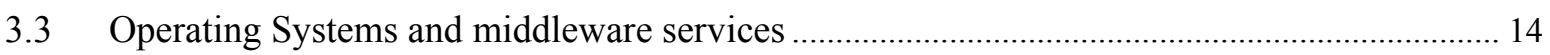

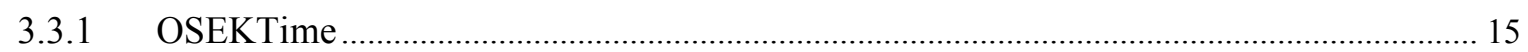

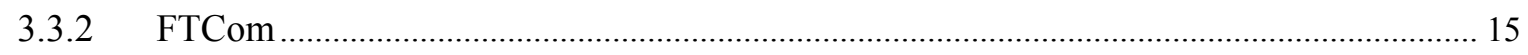

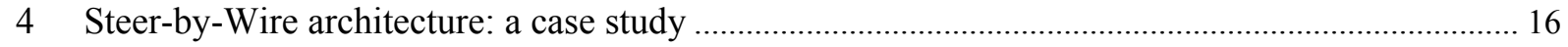

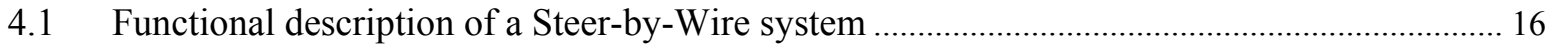

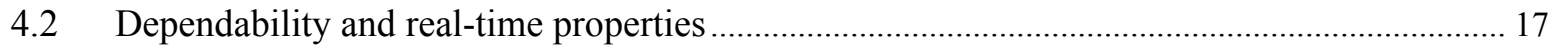

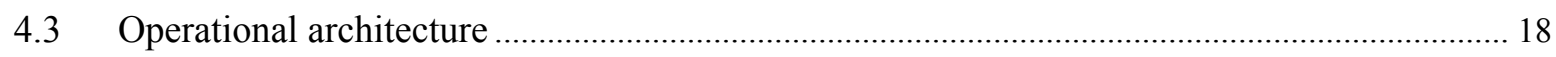

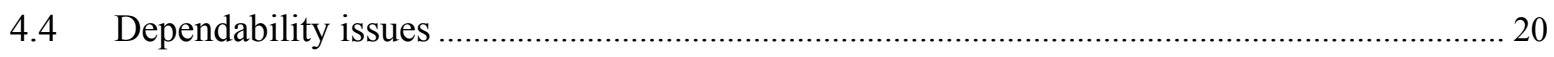

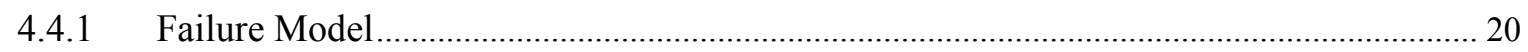

4.4.2 Operational architecture vs dependability requirements................................................ 22

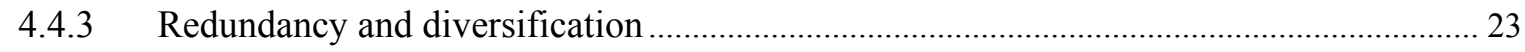

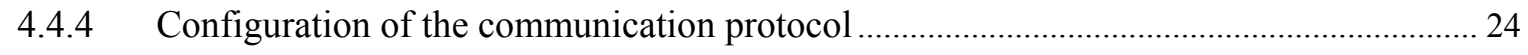

4.4.5 Evaluation of the Behavioral Reliability of the Architecture............................................... 26

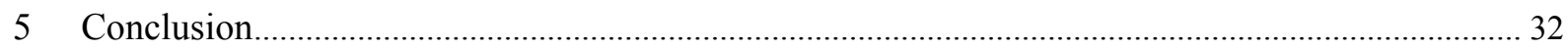

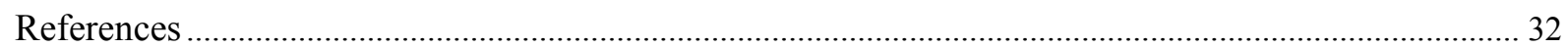

\section{Abstract :}

$\mathrm{X}$-by-Wire is a generic term referring to the replacement of mechanical or hydraulic systems, such as braking or steering, by electronic ones. In this chapter, we analyze the real-time and dependability 
constraints of X-by-Wire systems, review the fault-tolerant services that are needed and the communication protocols (TTP/C, FlexRay and TTCAN) considered for use in such systems. Using a Steer-by-Wire case-study, we detail the design principles and verification methods that can be used to ensure the stringent constraints of X-by-Wire systems.

\section{Why X-by-Wire systems ?}

Embedded electronics, and more precisely embedded software, is a fast growing area, and softwarebased systems are increasingly replacing the mechanical and / or hydraulic ones. The reasons for this evolution are technological as well as economical. On the one hand, the cost of hardware components is decreasing while their performances and reliability are increasing. On the other hand, electronic technology facilitates the introduction of new functions whose development would be costly, or not even feasible, if using mechanical or hydraulic systems alone. This evolution, formerly confined to functions such as motor control, wipers, lights, or door controls, now affects all car domains, even for critical functions such as throttle, brake or steering control. This trend resulted in the introduction of the concept "X-by-Wire", where mechanical or hydraulic systems embedded in an automotive application will be replaced by fully electric / electronic ones.

Historically, the first critical X-by-Wire function was the Throttle-by-Wire, implemented in a Chevrolet Corvette series in 1980 to replace the cable-based throttle. Today, this function is present in most vehicles, for example, the Peugeot 307. Shift-by-Wire systems, also known as Gear-by-Wire, are also implemented in some high end vehicles such as the BMW 5 and 7 series.

However, mechanical systems are still necessary for the most currently used X-by-Wire systems, either to work in conjunction with the electronic system or as a backup (examples: the Electronic Hydraulic Braking system, semi-active suspensions as in the Mercedes Adaptive Dampfung System, the Electronic Camshaft in the BMW Valvetronic technology, or the Robotized Gear Box). It is interesting to remark that the Robotized Gear Box is an option proposed by all carmakers in the world today.

One of the main obstacles for general acceptance of X-by-Wire systems is the difficulty to prove that all the necessary safety measures are followed. It is enough to note that the dysfunction of Steer-by- 
Wire, Brake-by-Wire or Throttle-by-Wire systems would jeopardize the safety of the occupants. As seen before, a number of X-by-Wire systems have already been implemented in certain series of vehicles, however, Steer-by-Wire and Brake-by-Wire systems will always have a mechanical backup. The concern for safety is certainly a major factor.

Another obstacle is that the customer's demand is not very great at the moment; he does not realize the technical advantages and only sees a higher price tag. But, the advantages of this technology can be very attractive for both carmakers and customers (see sections 1.1 and 1.2), which explains why carmakers are investing in this domain.

\subsection{Steer-by-Wire system}

The first advantage lies in the decreased risk of the steering column entering the cockpit in the event of a frontal crash. Furthermore, the variable steering ratio of the Steer-by-Wire system brings a remarkably increased comfort to the driver. This function enables the steering ratio between the hand wheel and the wheels to adapt according to the driving conditions. In parking and urban driving, this ratio should be smaller in order to reduce the amplitude of the hand wheel rotation. Another facility for steering functionality, brought by software-based technology, is the $\mu$-split braking which consists in applying a dissymmetric torque to the wheels in case of a dissymmetric adherence. Finally, the steering column is one of the heaviest components of the vehicle and removing it significantly decreases the weight of the vehicle and thus reduces fuel consumption.

Among the drawbacks, the electrical power needed to action the front axle requires the use of 42 Volt technology. At the SAE Conference in 2003 [1], it was said that this technology would not be mature before 2010. This announcement has considerably reduced the emphasis put on the X-by-Wire developments in general. Furthermore, the safety issues have not yet been fully defined.

\subsection{Brake-by-Wire systems}

A Brake-by-Wire system implemented with one micro-controller and one actuator per wheel can significantly increase the quality of the braking, in particular, by reducing the stopping distance. Moreover, this technology provides more precise braking by adapting to the pressure the driver puts 
on the pedal. Like Steer-by-Wire, there is a significant decrease in the weight of the vehicle in removing the hydraulic braking system and, therefore, significantly lowering costs. Finally, Brake-byWire will help to protect the environment because no braking fluid is necessary.

Unfortunately, as with the Steer-by-Wire system, the 42 Volt problem is a barrier for its present deployment. Another hindrance comes from the mentality of the customers. Indeed, the customer is faced with a new technology for a critical function that will be more expensive in the beginning and whose benefits he does not clearly see.

The first technology in Brake-by-Wire was the introduction of Electro Hydraulic Braking (EHB). The main difference between the EHB system and the classic braking system is that each wheel has an independent braking subsystem: the hydraulic pressure is applied independently on each wheel. However, a classic hydraulic circuit is still implemented from the pedal to the front wheels for safety reasons. The EHB system was factory installed for the first time in 2001 with the Mercedes Roadster SL. Today, Toyota proposes a "regenerative EHB" in their Prius, which uses the energy dissipated during deceleration to charge the battery.

\section{Problem, context and constraints for the design of X-by-Wire systems}

\subsection{General constraints}

As explained before, some X-by-Wire systems are already standard in certain series. However, while the implementation of Brake-by-Wire and Steer-by-Wire is possible with a 14 Volt battery in low weight vehicles, this is not the case for the heavier ones. As the first Brake-by-Wire and Steer-by-Wire will be costly in the beginning, they have greater chances of being implemented in high-end vehicles. Consequently, 42 Volt technology has to be mature before X-by-Wire systems can be mass produced. Moreover, Cell Fuel Technology, with fully electric energy sources, seems to be well situated for replacing combustion engines. This significantly reduces the necessity of 42 Volt technology in the long run [1].

In addition, the size of the X-by-Wire systems and their cost are major constraints for carmakers; electronics-based systems already account for $30 \%$ of the total cost in current vehicles. 


\subsection{Dependability constraints}

An analysis of the requirements for X-by-Wire systems was published in the conclusions of the X-byWire Esprit project 19]. In general, for a critical X-by-Wire system, it must be ensured that [19]:

- a system failure does not lead to a state in which human life, economics or environment are endangered,

- and a single failure of one component must not lead to a failure of the whole X-byWire system.

The system shall memorize intermittent failures and it shall signal a critical failure to the driver, for example through a lightning on the dashboard.. Moreover, it is required that the system is at least able to tolerate one major critical fault without loss of the functionality for a time long enough to reach a safe parking area. This requirement is a very constraining one, because if only two redundant components are used to provide a critical function, in case of failure of one of these components, the driver must immobilize the vehicle. This requirement will have to be confronted to the availability requirements in the future.

In terms of the criticality of the involved functions, automotive X-by-Wire systems can reasonably be compared with Flight-by-Wire systems in the avionic field. According to [19], the probability of encountering a critical safety failure shall not exceed $5 \cdot 10^{-10}$ per hour and per system, but other studies have been realized with a maximal bound of $10^{-9}$. This quantification can be translated in terms of Safety Integrity Level (SIL, see [2]) and a maximal bound of $10^{-9}$ corresponds to a SIL4 system. In fact, SIL4 conformance is reached below $10^{-8}$.

Up to now, it has been a challenge to reach such dependability because of the lack of experience in the automotive industry with X-by-Wire systems and because of the complexity of the problem. In particular, the environment (EMI, temperature peaks, ..) may reduce the predictability of the system and the design is subject to heavy cost and weight constraints. It is likely that, as in the aeronautic industry, for legal and technical reasons, the design process (e.g., software development, methods and tools for validation, ...) will have to be certified in the future. 
Finally, one objective that must be reached is that an X-by-Wire system offers the same availability and the same maintainability as their mechanical/hydraulic counterparts. The challenge is to prove that a given $\mathrm{X}$-by-Wire system adheres to all these requirements.

\subsection{Real-time constraints}

Belonging to the chassis domain, Steer-by-Wire and Brake-by-Wire systems are intrinsically real-time distributed systems. They implement complex multivariable control laws and deliver real-time information to intelligent devices that are physically distant (for example, the four wheels). They have to respect stringent time constraints, such as a sampling period of only a few milliseconds. End-to-end response times shall also be bounded, for example, the time between a request from the driver and the response of the physical system must be lower than a few tens of milliseconds (see the Steer-by-Wire example in section 4). An excessive end-to-end response time of a control loop may induce not only performance degradation but also cause the instability of the vehicle.

Although these constraints may differ according to the driving conditions, they all have to be respected whatever the situation. So, in general, the worst case scenario must be taken into consideration and these real-time constraints must be met with a high probability, even if the system is under random perturbations, because of the critical safety nature of the X-by-Wire applications.

\section{Fault-tolerant services for X-by-Wire}

\subsection{Overview on the communication services}

The communication system has to provide services that are pertinent with respect to the dependability objectives of the application (see section 2). For instance, the knowledge of the stations that are operational at a given time is usually needed by X-by-Wire applications and thus, a membership service that furnishes this information will ease the development of application-level software and its accuracy. 


\subsubsection{Time-Triggered communication}

Among communication networks, one distinguishes time-triggered protocols where activities are driven by the progress of time and event-triggered protocols where activities are driven by the occurrence of events. Both types of communication have advantages but one considers that, in general, dependability is much easier to ensure using a time-triggered bus (refer, for instance, to [3] for a discussion on this topic). This explains that, currently, only time-triggered communication systems are being considered for use in X-by-Wire applications. In this category, multi-access protocols based on TDMA (Time Division Multiple Access) are particularly well suited; they provide deterministic access to the medium (the order of the transmissions is defined statically at the design time and organized in "rounds"), and thus bounded response times. Moreover, their regular message transmissions can be used as "heartbeats" for detecting station failures.

The two TDMA based networks that are candidates for supporting X-by-Wire applications are TTP/C (see 3.2.1) and FlexRay (see 3.2.2). At the time of writing, FlexRay, which is backed by the major players of the European automotive industry, seems in a strong position for becoming the standard in the industry, although the specifications are not yet finalized.

\subsubsection{Fault Tolerant Unit (FTU)}

To achieve fault-tolerance, that is to say, the capacity of a system to deliver its service even in the presence of faults, certain nodes are replicated and clustered into Fault-Tolerant Units (FTUs). An FTU is a set of several stations which perform the same function and each node of an FTU possesses its own slot in the round so that the failure of one or more stations in the same FTU can be tolerated. Actually, the role of FTUs is two-fold. First, they make the system resilient in the presence of transmission errors (some frames of the FTU may still be correct while others are corrupted). Second, they provide a means to fight against measurement and computation errors occurring before transmission (some nodes may send the correct values while others may make errors). The stations forming an FTU will be called replicas from here out. The use of fail-silent nodes (see section 3.1.3) greatly simplifies the design of a fault-tolerant architecture. However the fail-silent property is generally not easy to verify. The number of replicas per FTU, which are required to tolerate k faulty 
components, depend on the behavior of the individual components (see [4]). For instance, if the failure of $\mathrm{k}$ nodes must be tolerated, then the least necessary number of replicated nodes is $k+1$ when all nodes are fail-silent.

\subsubsection{Fail-Silent Node}

As previously explained, fail-silent nodes greatly decrease the complexity of the design of a critical application. A node is said fail-silent if:

1. a) it sends frames at the correct point in time (correctness in the time domain), and b) the correct value is transmitted (correctness in the value domain), or

2. it sends detectably incorrect frames (e.g., wrong Cyclic Redundancy Check - CRC) in its own slot or no frame at all

A communication system, such as TTP/C, is able to provide very reliable support for the requirements 1.a) and 2) (which provide the so-called "fail-silence in the temporal domain" once they are accomplished), especially through the bus guardian concept, while the value domain is mainly the responsibility of the application. The reader is referred to $[4,5,6]$ for good starting points on the difficult problem of ensuring fail-silence

\subsubsection{Bus Guardian}

When communications are multiplexed, it may happen that a faulty ECU, transmitting outside its specification, for instance, at the wrong time or with a larger frame, perturbs the correct functioning of the whole network. One well-known manifestation is the so-called "babbling idiots" nodes that transmits continuously. To avoid this situation, a component called the "bus guardian", restricts the controller's ability to transmit by allowing transmission only when the node exhibits a specified behavior. Ideally, the bus guardian should have its own copy of the schedule, it should be physically separated from the controller, it should possess its own power supply and it should be able to construct the global time itself. Due to the strong pressure from the automotive industry concerning costs, these assumptions are not fulfilled in general, which reduces the efficiency of the bus guardian strategy. 


\subsection{Main time-triggered protocols for automotive industry}

\subsubsection{TTP/C}

The TTP/C protocol (Time Triggered Protocol), which is defined in [7], is a central part of the TimeTriggered Architecture (TTA - see [8]) and it possesses numerous features and services related to dependability such as the bus guardian (see [5]), the group membership algorithm (see [9]) and support for mode changes (see [10]). The TTA and the TTP/C protocols have been designed and extensively studied at the Vienna University of Technology. Hardware implementations of the TTP/C protocol, as well as software tools for the design of the application, are commercialized by the TTTech company [11] and available today.

On a TTP/C network, the transmission support is replicated and each channel transports its own copy of the same message. Although an Electro Magnetic Interference (EMI) is likely to affect both channels in quite a similar manner, the redundancy provides some resilience to transmission errors (see section 4.4.4) TTP/C can be implemented with a bus topology or a star topology. The latter topology provides better fault tolerance since the star can act as a central bus guardian and protect against errors that cannot be avoided by a local bus guardian such as spatial proximity fault. For instance, a star topology is more resilient to spatial proximity faults and to faults due to a desynchronization of an ECU (see section 3.1.4). To avoid a single point of failure, a dual star topology shall be used with the drawback that the length of the cables is significantly increased.

At the Medium Access (MAC) level, the TTP/C protocol implements the synchronous TDMA scheme: the stations (or nodes) have access to the bus in a strict deterministic sequential order and each station possesses the bus for a constant period of time called a "slot" during which it has to transmit one frame. The sequence of slots such that all stations have accessed the bus one time, is called a "TDMA round". An example of a round is shown in Figure. The size of the slot is not necessarily identical for all stations in the TDMA round, but successive slots belonging to the same station are of the same size. Consecutive TDMA rounds may differ by the data transmitted during the slots, and the sequence of all TDMA rounds is the "cluster cycle" which repeats itself in a cycle. 
TTP/C includes powerful but complex algorithms for easing and speeding up the design of faulttolerant applications and some of them have been formally verified (see for instance [9] and [12]). In particular, TTP/C implements a clique avoidance algorithm and a membership algorithm that also provides data acknowledgment. The fault hypothesis used for the design of TTP/C is well specified and also quite restrictive (two successive faults must occur at least two rounds apart). Situations outside the fault hypothesis are treated using "never give up" (NUP) strategies [3] which aim to continue operation in a degredaded mode. For example, an usual method is that each node switches to local control according to the information still available, while trying to return to the normal mode.

\subsubsection{FlexRay}

A consortium of major companies from the automotive field is currently developing the FlexRay protocol. The core members are BMW, Bosch, DaimlerChrysler, General Motors, Motorola, Philips and Volkswagen. The specifications of the FlexRay Protocol are not publicly available nor finalized at the time of writing, however, material describing the protocol is available on the FlexRay web site $[13]$.

The FlexRay network is very flexible with regard to topology and transmission support redundancy. It can be configured as a bus, a star or multi-stars and it is not mandatory that each station possess replicated channels, even though this should be the case for X-by-Wire functions.

At the MAC level, FlexRay defines a communication cycle as the concatenation of a time-triggered (or static) window and an event triggered (or dynamic) window. In each communication window, whose size is set statically at design time, a different protocol is applied. The communication cycles are executed periodically. The time-triggered window uses a TDMA MAC protocol; the main difference with TTP/C is that a station might possess several slots in the time-triggered window, but the size of all slots is identical. In the event-triggered part of the communication cycle, the protocol is FTDMA (Flexible Time Division Multiple Access): the time is divided into so-called mini-slots, each station possesses a given number of mini-slots (not necessarily consecutive) and it can start the transmission of a frame inside each of its own mini-slots. A mini-slot remains idle if the station has 
nothing to transmit. An example of dynamic window is shown in

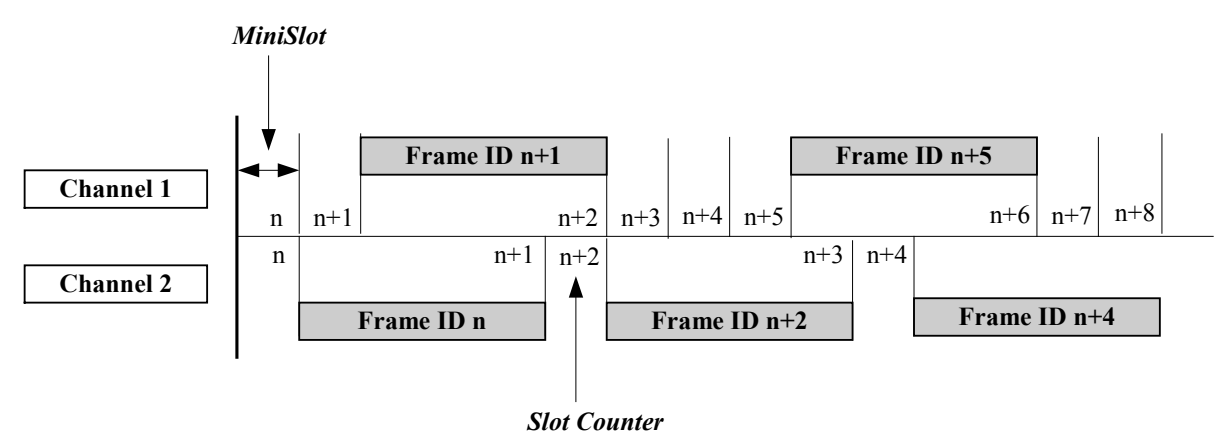

Figure 2: on channel $A$, frame $m$ started to be transmitted in mini-slot $m$ while mini-slots $m+1$ and $m+2$ have not been used. It is noteworthy that the frame $m+3$ is not received simultaneously on channels A and B since, in the dynamic window, transmissions are independent on both channels. The FlexRay MAC protocol is much more flexible than the TTP/C MAC since in the static window nodes are assigned as much slots as necessary (up to 4095 for each node) and since frames are only transmitted if necessary in the dynamic part of the communication cycle. Compared to TTP/C, the structure of the communication cycle is not statically stored in the nodes, it is indeed revealed during the startup phase. However, unlike TTP/C, mode changes with a different communication schedule for each mode are not possible.

TDMA

Static Segment
FTDMA

Dynamic Segment

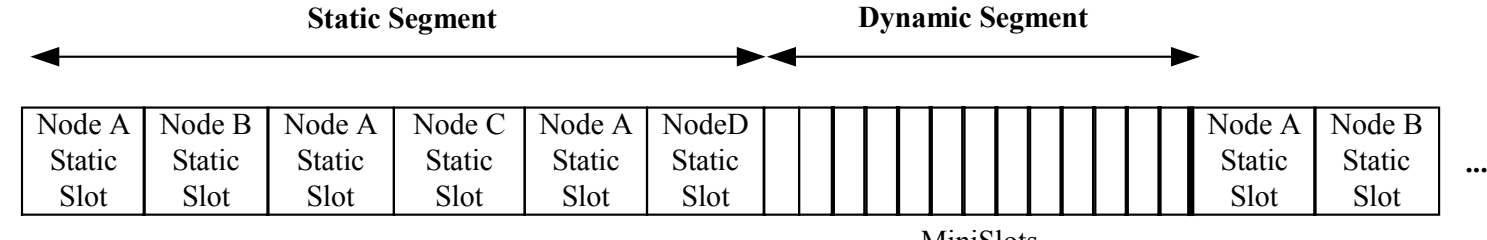

MiniSlots

Figure 1: Example of a FlexRay communication cycle with 4 nodes A, B, C and D

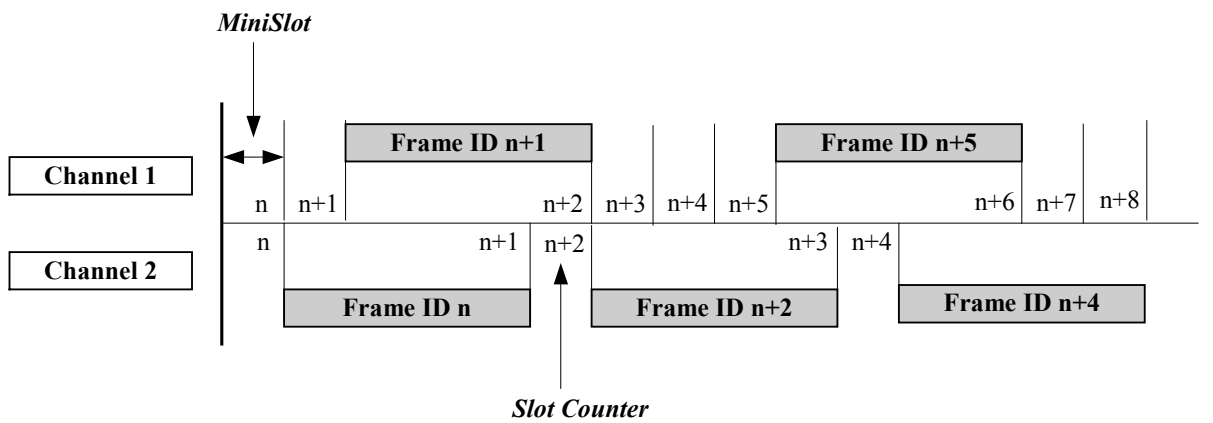


Figure 2: Example of message scheduling in the dynamic segment of the FlexRay communication cycle

From the dependability point of view, all services and functionalities of FlexRay, except the bus guardian and the clock synchronization, are not currently well documented nor is the fault-hypothesis used for the design. However, it seems that most features will have to be implemented in software or hardware layers on the top of FlexRay with the drawback that efficient implementations might be more difficult to achieve.

\subsubsection{TTCAN}

TTCAN (Time Triggered Controller Area Network - see [14]) is a communication protocol developed by Robert Bosch GmbH on the basis of the CAN physical and Data-Link Layer. Time-triggered communication is built upon the standard CAN protocol but the controllers must be able to disable automatic retransmission and provide the application with the time at which the first bit of a frame was sent or received [15].

The bus topology of the network, the characteristics of the transmission support, the frame format, as well as the maximum data rate - $1 \mathrm{Mbits} / \mathrm{s}$ - are imposed by CAN protocol. Channel redundancy is possible, but not standardized and no bus guardian is implemented in the node. A key idea is to propose, as with FlexRay, a flexible time-triggered/event-triggered protocol. TTCAN defines a basic cycle (the equivalent of the FlexRay communication cycle) as the concatenation of one or several time-triggered (or "exclusive") windows and one event-triggered (or "arbitrating") window. Exclusive windows are devoted to time triggered transmissions (i.e., periodic messages) while the arbitrating window is ruled by the standard CAN protocol: transmissions are dynamic and bus access is granted according to the priority of the frames. Several basic cycles that differ by their organization in exclusive and arbitrating windows and by the messages sent inside exclusive windows can be defined. The list of successive basic cycles is called the system matrix and the matrix is executed in loops. Interestingly, the protocol enables the master node, the node that initiates the basic cycle through the transmission of the "reference message", to stop the functioning in TTCAN mode and to resume in 
standard CAN. Later, the master node can switch back to TTCAN mode by sending a reference message.

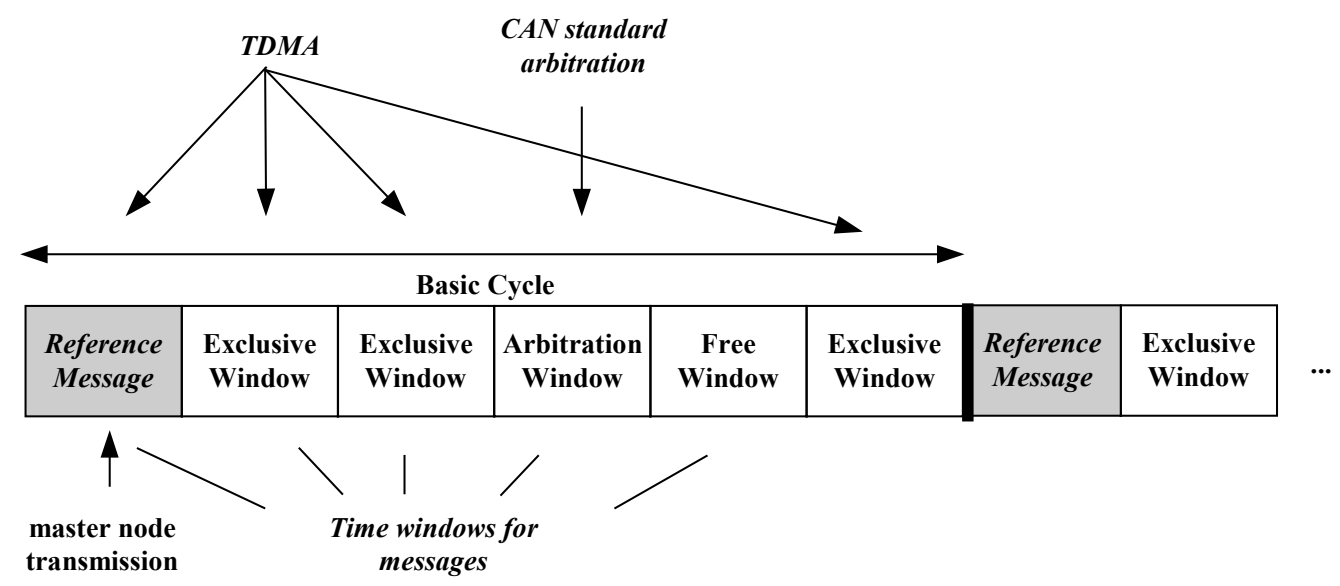

Figure 3: Example of a TTCAN Basic Cycle

TTCAN is built on a well-mastered and cheap technology which is CAN but, as defined by the standard, does not provide important dependability services such as the bus guardian, membership service and reliable acknowledgement. It is, of course, possible to implement some of these mechanisms at the application or middleware level but with a reduced efficiency. It seems that carmakers may consider the use of TTCAN for some systems during a transition period until the FlexRay technology is fully mature.

\subsection{Operating Systems and middleware services}

In the context of automotive applications, a middleware is a software layer located above the platform (hardware, operating system, protocols) that aims to offer high-level services to the application in order to reduce the time needed to market and improve the overall quality of the system. The main purpose of a middleware is to hide the distribution of the functions and the heterogeneity inside the platform (ECU, network, CPU, OS, ...). Another interest of middleware is to provide high level services; and, for X-by-Wire applications, services related to dependability are needed.

Several projects aimed at the development of automotive middleware layers have been undertaken (EAST: www.east-eea.net, AUTOSAR: http://www.autosar.org/). To the best of our knowledge, the only results publicly available have been produced in the context of the OSEK/VDX consortium (detailed information can be obtained at http://www.osek-vdx.org), which is a project of the 
automotive industry whose objective is to build a standard architecture for in-vehicle control units. Among the results of the OSEK/VDX group, two are of particular interest for X-by-Wire: the OSEKTime operating and the Fault-Tolerant Communication layer.

\subsubsection{OSEKTime}

OSEKTime OS (OSEK/VDX Time-Triggered Operating Systems, see [16]) is a small operating system designed to offer the basic services needed by time-triggered applications. In particular, OSEKTime OS offers services for task management, interrupt processing and error handling. An offline-generated dispatcher table, termed a "dispatcher round", activates the tasks in a determined order and repeats itself as long as the system is running. Several different dispatcher tables, corresponding, for instance, to different functioning modes of the system, can be defined, but the switching from one table to the next can only take place at the end of a round.

Tasks cannot be blocked waiting for an external event but they can be preempted; a running task will always be preempted by a task that is activated and the designer must take resource contention into account in this case. An interesting feature of OSEKTime OS is the deadline monitoring that can be performed for some specified tasks: when such a task is still not finished at its deadline, a specific application error handling routine is invoked and the operating system is re-initialized. In OSEKTime OS, the rate at which interrupts can occur is bounded, at a rate specified when the system is configured, in order to keep the system predictable.

As for communication cycles in time-triggered networks, the configuration of the dispatcher round can be done off-line through a software tool that will ensure the correctness of the system.

\subsubsection{FTCom}

OSEK/VDX FTCom (Fault-Tolerant Communication, see [17]]) is a proposal for a software layer that provides services for facilitating the development of fault-tolerant applications on top of timetriggered networks. One important function of FTCom, with respect to X-by-Wire, is to manage the redundancy of data needed for achieving fault-tolerance (see section 3.1.2). From an implementation point of view, it is sometimes preferable to present only one copy of data to the application in order to 
simplify the application code and to keep it independent from the level of redundancy (i.e., the number of nodes composing an FTU). In OSEK/VDX terminology, the algorithm responsible for the choice of the value that will be transmitted to the application is termed "the agreement algorithm". Many agreement strategies are possible: pick-any (fail-silent node), average-value, pick-a-particular-one, majority vote, etc. OSEK FTCom provides a generic way for specifying the agreement strategy of replicated data.

Two other important services of the FTCom are 1) to manage the packing/unpacking of messages [18], which is needed if the use of network bandwidth has to be optimized, and 2) to provide message filtering mechanisms for passing only "significant" data to the application.

\section{Steer-by-Wire architecture: a case study}

A Steer-by-Wire system aims to provide two main services: controlling the wheel direction according to the driver's request and providing a "mechanical-like" force feedback to the hand wheel. In this section, we present the functional point of view of such a system, the real-time and dependability properties that have to be observed, and a realistic operational Steer-by-Wire system used as a reference architecture for evaluation purposes. Finally, we will focus on the real-time requirements and, after proposing a way to model failures, we will show how each component or subsystem of the reference architecture can reach the dependability objective.

\subsection{Functional description of a Steer-by-Wire system}

In a Steer-by-Wire system, two main services have to be provided: the front axle actuation and the hand wheel force feedback. So, from a functional point of view, this implies two main functions that are not completely independent. However, in the following discussion, in order to simplify their description, we will not take into account the interdependencies between these functions.

Front axle control. This function computes the orders that are given to the motor of the front axle, mainly according to the state of this front axle and the commands given by the driver through the hand wheel. The driver's requests are translated through:

- the hand wheel angle, 
- the hand wheel torque,

- the hand wheel speed.

Hand wheel force feedback. This function computes the order that will be given to the hand wheel motor, in particular, according to:

- the speed of the vehicle,

- the front axle position,

- the front tie rod force.

The elaboration of these orders requires the execution of filtering algorithms, complex control laws under stringent sampling periods of a few milliseconds. The main property to ensure is that the end-toend response time between a new command from the driver and the effect on the front axle is bounded.

\subsection{Dependability and real-time properties}

As stated in section 2.2, a Steer-by-Wire system must comply with the Safety Integrity Level 4 (SIL4 [2]). This means that the system shall be able to tolerate a single failure and to ensure that the probability of encountering a safety-critical failure does not exceed $10^{-9}$ per hour. An important issue is to formally determine the relation between the distributed system that supports the steering control function and the failure occurrences at the steering system level. Section 4.4.5 aims to propose a few solutions to this problem.

Besides these dependability constraints, any steering system has to ensure certain performances. Specifically, let us consider the front axle control function. According to the vehicle technical requirements stated in [19], whatever the system is (i.e., mechanical / hydraulic or by-wire), the maximum angles of the front wheels should be at least \pm 40 degrees (respectively, \pm 90 degrees for upcoming systems). This leads to a specific performance property imposing the ability to control the steering with a velocity of at least 40 degrees per second (respectively, 90 degrees).

Furthermore, some real time properties are derived from control requirements. In particular, some control laws implemented in the case study required a sampling period equal to $2 \mathrm{~ms}$ (i.e., 500 sampled wheel angle values per second). Each sample was treated through filtering and control 
algorithms and led to a value that is used by an actuator in order to reach the desired steering position. Obviously a delay (named end-to-end response time), that cannot be neglected, exists between one sampling and its corresponding reaction on the actuator. This delay is mainly due to the execution of the algorithm on the ECU and to the transfer of the sampled value through the communication systems. In order to guarantee vehicle stability, this delay has to be less than a given bound that depends on the type of vehicle as well as on the driving condition (velocity, wheel angle, ...). It is the carmakers' responsibility to be able to compute the limit of the tolerated delay for any given situation the vehicle may be in.

Notice that the occasional absence of samples or out-of-bound delays at the controller or actuator level, for instance, due to frame loss, does not necessarily lead to vehicle instability, but degrades steering performance (or Quality of Service). This is because most of the control laws that are used are designed with specific delay and/or absence of sampling data compensation mechanisms, tolerating thus, perturbations under a given threshold. In section 4.4.5.1, we will show an example of how to evaluate such a threshold value using a Matlab/Simulink model of the system.

\subsection{Operational architecture}

An operational architecture, which is a solution for the implementation of the functions presented in section 4.1, is described in this section. Figure 4 illustrates the hardware architecture on which the operational one is based. It includes four Electronic Control Units (micro-controllers) named, respectively, HW ECU1 (Hand Wheel ECU1), HW ECU2 (Hand Wheel ECU2), FAA ECU1 (Front Axle Actuator ECU1), and FAA ECU2 (Front Axle Actuator ECU2). Each node is connected to the two TDMA-based communication channels (BUS1 and BUS2). Finally, three sensors, named as1, as2 and as3, placed near the hand wheel measure the requests of the driver in a similar way, the latter being translated into a 3-tuple $<$ hand wheel angle, hand wheel torque, hand wheel speed $>$. Three other sensors, named rps1, rps2 and rps3, are dedicated to the measurement of the front axle position. Finally, two motors (FAA Motor2 and FAA Motor2), configured in active redundancy, act on the front axle while two other motors (HW Motor1 and HW Motor2) realize the force feedback control on the 
hand wheel. Sensors as1, as2 and as3 (respectively, sensors rps1, rps2 and rps3) are connected by point-to-point links, both to HW ECU1 and HW ECU2 (respectively, FAA ECU1 and FAA ECU2).

\section{Implementation of the "Front axle control" function}

The requests of the driver are measured by the three replicated sensors as 1 , as 2 and as 3 and sent to both HW ECU1 and HW ECU2. Each ECU performs a majority vote on the 3 received values and transmits a "secure" data on both communication channels BUS1 and BUS2. The two ECUs, FAA ECU1 and FAA ECU2, placed behind the front axle, consume this data, as well as the last wheel position, in order to elaborate the commands that are to be applied to FAA Motor 1 and 2.

\section{Implementation of the "Force feedback control" function}

In a way similar to the previous function, measurements taken by rps1, rps 2 and $\operatorname{rps} 3$ are transmitted both to FAA ECU1 and FAA ECU2. Each of these ECUs elaborates information transmitted on the network. The consumers of this information are both HW ECU1 and HW ECU2 which compute the command transmitted to HW Motor 1 and 2.

The replication of algorithms on several ECUs, of measurements from several similar sensors and of information transmission over redundant buses is highly used in this operational architecture. The choices made in terms of redundancy and diversification (see section 4.4.3) are constrained by dependability, cost and dimension requirements. Alternative Steer-by-Wire architectures presented in literature (e.g., [19]) use, in addition, two central ECUs located between the hand wheel and the front but, from an economic point of view, it is of course preferable to use only four ECUs if dependability criteria are met. 


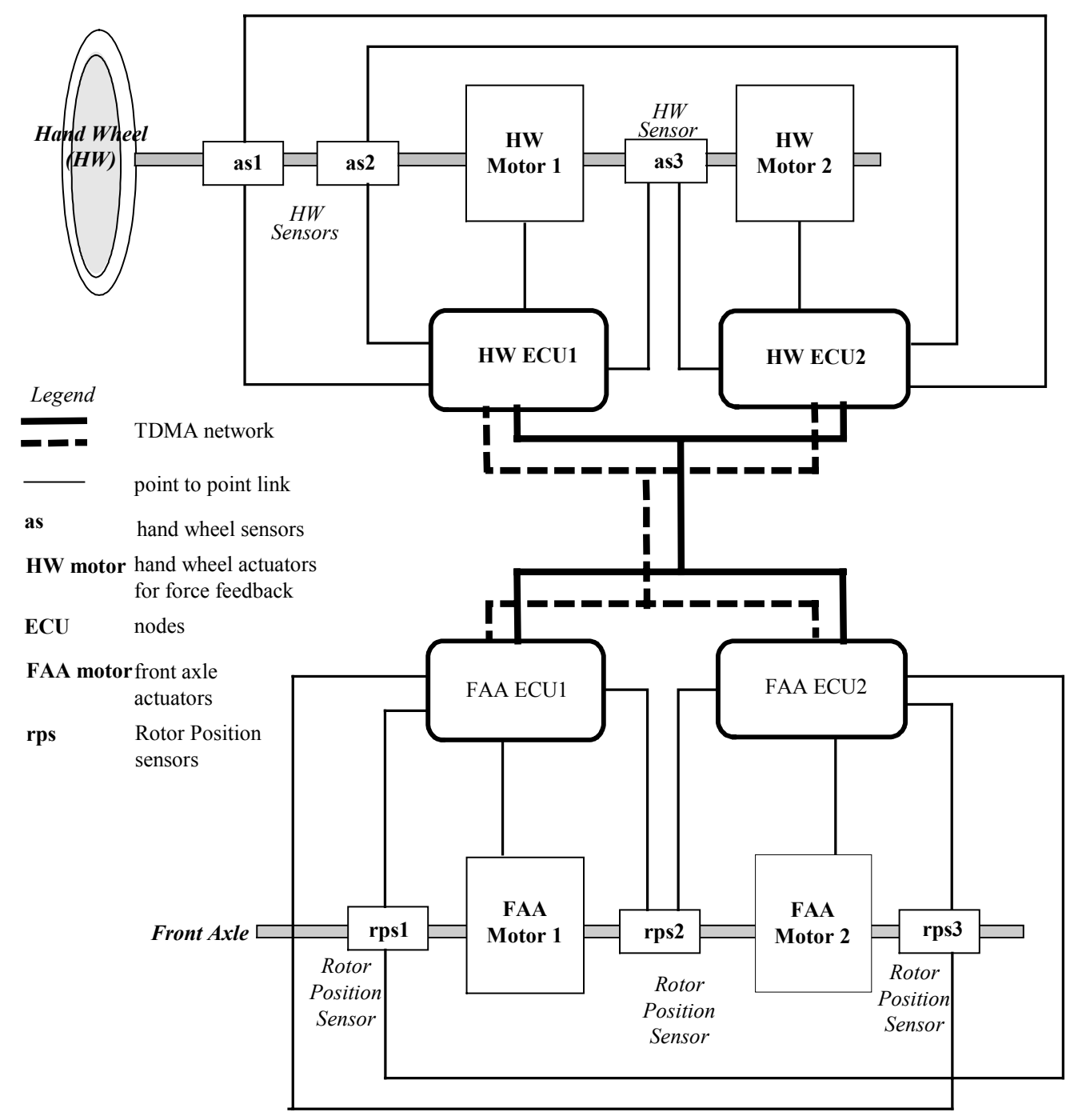

Figure 4 : Steer-by-Wire operational architecture

\subsection{Dependability issues}

In this section, after recalling which failures are taken into account, we justify choices that are made for the specification of the hardware architecture.

\subsubsection{Failure Model}

The terms fault, error and failure are currently used in system engineering. In [20], these terms are clearly defined. Let us consider that a system has to deliver a service. A system failure is an event that occurs when the delivered service deviates from the expected one. An error is the part of the state of the system that may cause a subsequent failure and a fault is the adjudged cause of an error. A fault is active when it produces an error, otherwise it is dormant. Note that, if we consider that a system is 
composed of components, we can observe possible causal relations between failure of one or several components and failure of the system:

$$
\frac{\text { Component }}{\text { Fault } \rightarrow \text { Error } \rightarrow \text { Failure }} \rightarrow \frac{\text { System }}{\text { Fault } \rightarrow \text { Error } \rightarrow \text { Failure }}
$$

Figure 5: Failure propagation

Usually, two classes of faults can be distinguished according to their effects inside a system: Byzantine faults and coherent faults. These faults are caused by a failure of one or several components of the system. A Byzantine fault is a fault whose effect can be perceived differently by different observers. The effect of a coherent fault is seen the same by all observers. Moreover, the property of "fail silence", assumed for some components, leads to a third class of fault. A component is said to be "fail silent" if, each time it is not silent, we can conclude that it is functioning properly. Note that at a system level, the silence of this class of component is seen as a fault when it occurs.

A second classification of faults relies on the duration of a fault. In this case, we consider two types of faults according to their effect on the whole system. In our context, we will state that "transient fault" is a fault whose duration is such that the system does not reach a "not safe state". A "permanent fault" is a non-transient one. The main issue is to evaluate the delay after which a transient fault becomes a permanent one. In section 4.4.5.1, we present a method for the evaluation of the "worst tolerable delay".

Following the approach proposed in [21], we define the system dependability requirement by a triplet $\operatorname{FM}(b, c, f)$, named "Flexible Failure Model", where b is the maximum number of Byzantine failing sources, $\mathrm{c}$ the maximum number of coherent failing sources, and $\mathrm{f}$ the maximum number of fail-silent sources that the system must be able to tolerate. In this case study, we consider a failure model defined by $\operatorname{FM}(1,1,1)$ : the system must always tolerate, at a given time, one Byzantine fault or one coherent fault or a fault due to one fail-silence of a component. 


\subsubsection{Operational architecture vs dependability requirements}

In this section, we give some rules that we applied for designing the architecture. The system under study has to provide two main services: controlling the front axle according to the driver requests and furnishing a force feedback to the driver. We focus on the former. A similar rationale can be used for the second one. In both cases, the main question is the evaluation of the minimum number of redundant components that contribute to meet the dependability requirement.

Dependability analyses are generally based on a strong hypothesis assuming that, in the whole system, $n$ simultaneous component failures can never occur for any set of redundant components (set of redundant hand wheel sensors, set of redundant "Hand Wheel ECU", ...). In this case study, we suppose that $n$ is equal to 1 .

Note that in [22], Lamport states that $3 n+1$ redundant components are necessary to tolerate $n$ Byzantine faults. In order to tolerate $n$ coherent faults, it is sufficient to have $2 n+1$ redundant components.

\section{ECU redundancy}

Two functions need to be implemented in ECU: Front Axle Control and Force Feedback Control. To avoid costly and numerous wires, ECUs have to be placed close to the sensors, and communication between ECUs has to be multiplexed. A Hand Wheel ECU (respectively, a Front Axle ECU) is a consumer of information sampled from the hand wheel (respectively, front axle) and is a producer of information used by the Front Axle Control function (respectively, Force Feedback Control function) implemented in a Front Axle ECU (respectively, in a Hand Wheel ECU). According to the rule given by Lamport, the minimum number of redundant Hand Wheel ECUs (respectively, Front Axle ECUs) should be 4 . This solution is mainly used in the aeronautic domain. But, automotive requirements are completely different in terms of cost and space. Therefore a classical solution is to use Fail-Silent ECUs. In this case, obviously, only two Hand Wheel ECUs (respectively, Front Axle ECUs) are necessary. However, we have to ensure the fail-silence property. To do this, several techniques based on the Petri-Nets analysis [23], C Model simulation [24] or fault injection [25] are used. 


\section{Hand Wheel Sensor redundancy}

A Hand Wheel sensor produces information for two Hand Wheel ECUs. Three Hand Wheel sensors are necessary for ensuring that each Hand Wheel ECU, assumed to provide a voting algorithm, is able to tolerate one Byzantine fault (and subsequently one coherent fault or one fail-silent sensor).

\section{Actuator Redundancy}

Actuators are mechanical components without any calculating ability, and a single actuator can take charge of piloting the Front Axle. Furthermore, according to the inherent reliability properties of these actuators, we guarantee that an actuator can never wrongly apply an order received by a Front Axle ECU. Under these assumptions and taking into account the formerly stated fail-silence property of a Front Axle ECU, only 2 couples $<$ Front Axle ECU,actuator $>$ are necessary for the tolerance of, at most, one fault.

\subsubsection{Redundancy and diversification}

According to the dependability requirements, presented in section 4.2, and to the assumption made on the ECUs (fail-silence in our case) and according to the failure occurrence model (section 4.4.1), a certain level of redundancy has to be implemented. If the chosen fault-tolerance strategy is failure recovery, redundant ECUs will work only in the case of the primary ECU failing. In this case, failure detection must be quick and reliable. Otherwise, if the strategy is failure compensation, as with the front axle motors, redundant ECUs will be placed in parallel and work simultaneously. Because of the stringent real-time constraint, our architecture must provide failure compensation.

It is worthy to note that the redundancy of identical ECUs does not prevent the architecture from common mode failures: the hardware of redundant ECUs should be furnished by different suppliers and their software realized by different teams. The Ariane 501 explosion is a good example to show the importance of diversification. Both back-up and active inertial reference systems failed for the same reason [26]. If software and/or hardware had been diversified, one of the two inertial reference systems would have remained safe. But, for cost and maintenance reasons, it is not always possible to implement diversified components and technologies. 


\subsubsection{Configuration of the communication protocol}

Communication is driven by a TDMA-based protocol with two replicated channels. More precisely, the network that is used in this case study is TTP/C because of the availability of the protocol specification and the components. However, the same analysis is valid for any time-triggered protocol such as TTCAN and FlexRay.

For reliability reasons, the same frame is transmitted on the two replicated channels. In order to avoid common mode failures (EMI, temperature, ...) channels should be placed as far as possible from each other in the vehicle.

\subsubsection{Slot allocation strategy to maximize the "robustness" of the transmission}

In TTP/C, the transmission order inside a round can be freely chosen by the application designer. Among the criteria for constructing the TDMA round, applicative constraints like computation time and sampling rates can be taken into account. But, as shown in $[27,28]$, the robustness of a TDMAbased system against transmission errors heavily depends on the location of the slots inside the round. In automotive systems, one observes that transmission errors are highly correlated: there occur perturbations that corrupt several consecutive frames (so called "bursts" of errors). Should two frames that belong to the same FTU (Fault Tolerant Unit, see section 3.1.2) be transmitted just one after the other, then a single perturbation could corrupt both of the frames. The objective to pursue depends on the status of the FTU with regard to the concept of "fail-silence" (see 3.1.3). For FTUs composed of a set of fail-silent nodes, the successful transmission of one single frame for the whole set of replicas is sufficient since the value carried by the frame is necessarily correct. In this case, the objective to achieve with regard to the robustness against transmission errors is the minimizing of the probability that all frames of the FTU (carrying data corresponding to the same production cycle) be corrupted. This probability is denoted $P \_$all in [27].

In practice, replicated sensors may return slightly different observations and, without extra communication for an agreement, replicated nodes of a same FTU may transmit different data. If a decision, such as a majority vote, has to be taken by a node with regard to the value of the transmitted data, the objective is to maximize probability that at least one frame of each FTU is successfully 
transmitted during a production cycle. If the production cycle is equal to one round then it comes back to minimizing $P_{\text {_one }}$, the probability that one or more frames of an FTU have become corrupted.

It has been shown in [27], with some reasonable assumptions on the error model, that the optimal

solution to minimize $P_{-}$all is to "spread" the different frames of a same FTU uniformly over the TDMA round. An algorithm that ensures the optimal solution is provided for the case where the FTUs have, at most, two different cardinalities (for instance, one FTU is made of 2 replicas and other FTUs are made of 3 replicas). For the other cases, a low-complexity heuristic is proposed [27], and it was proven to be close to the optimal on simulations that were performed.

In [28], it was demonstrated that, under very weak assumptions on the error model and whatever the number of FTUs and their cardinalities, the clustering together of the transmission of all the frames of an FTU minimizes $P \_$one when the production cycle of the data sent is equal to the length of a TDMA round.

These two results, for the fail-silent case and non fail-silent case, provide simple guidelines to the application designer for designing the schedule of transmission. In our case-study, since all ECUs are fail-silent, our requirement is to minimize the probability of losing all replicas in the TDMA round, and thus the redundant frames have to be spread over time.

\subsubsection{Allocation of the slots in the round}

Let us consider the architecture shown in Figure 4 with the following characteristics for the production of data:

HW ECU1/ HW ECU2 - production of two pieces of data packed in a single frame:

- HW Angle every 2ms,

- $\quad$ HW Torque every $4 \mathrm{~ms}$.

FAA ECU1/ FAA ECU2 - production of two pieces of data packed in a single frame:

- FAA position every $2 \mathrm{~ms}$,

- $\quad$ Tie Rod Force every $4 \mathrm{~ms}$.

The size of the TDMA Round is set to the minimal production period, i.e., $2 \mathrm{~ms}$. Since the frames are composed of the same information, whatever the round, the size of the cluster cycle is equal to one 
round, which is possible with the latest version of the specification [7]. According to all these considerations, the location of the slots inside the round will be the following:

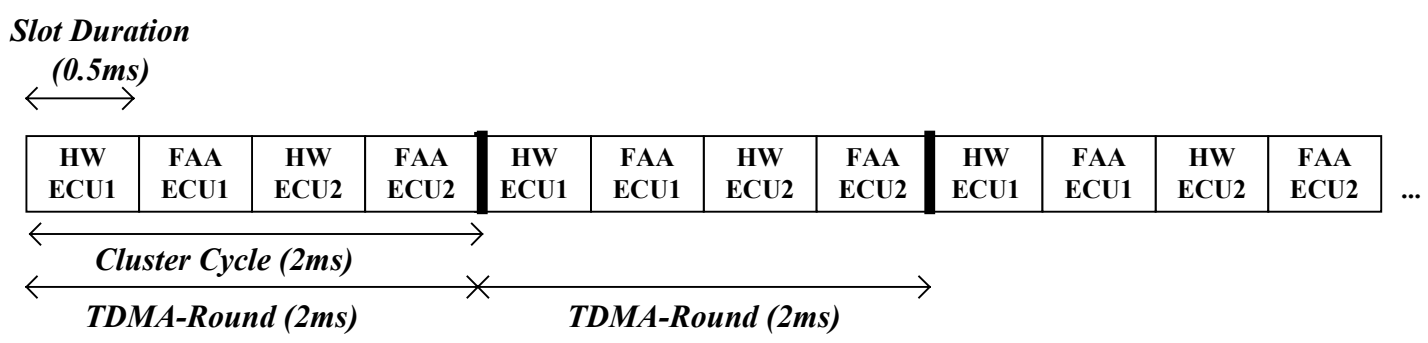

Figure 6: Placement of the slots in the cluster cycle

The slot duration is set equal for every slot. However, it is not a constraint imposed by the TTP/C protocol but, in this case study, this choice has been justified by application level constraints (deadline on tasks, ...).

\subsubsection{Evaluation of the Behavioral Reliability of the Architecture}

For a given Mean Time To Failure (MTTF) of the components (sensors, computers, network links, actuators) and their redundancy, the use of classic reliability analysis methods, for instance Fault-Tree Analysis (FTA) and Failure Mode and Effect Analysis (FMEA), can provide an estimation of the reliability of a Steer-by-Wire architecture (see, for instance, [29]). However, neither transient faults nor real-time performances are taken into account in these kinds of evaluation, and thus, these evaluations are clearly not sufficient in our context.

Under normal conditions, the use of a time-triggered scheduling of tasks and messages allows the reception of the sensor data at regular intervals, thus providing bounded end-to-end delays. However, random environmental perturbations (e.g., EMI) could make the communication system unavailable during some time periods. For example, consecutive transmission errors can create a time period during which the controller or the actuators do not receive any sensor data. The concept of behavioral reliability, defined in [30], for determining the probability with which the Steer-by-Wire system violates the end-to-end delay constraints for one hour under a stochastic perturbation model. Our objective is to ensure this probability must be less than $10^{-9}$ which is more stringent than the SIL4 requirement, see section 2.2 . 
The end-to-end delay for the front axle control function is composed of the so-called "pure delay" (delay induced by the system before the driver's command is given to the actuators) and the mechatronic delay introduced by the actuators (electric motors in our case). The mechatronic delay can be bounded by a constant $T_{m e c}$. In what follows, we will only focus on the analysis of the pure delay analysis which is denoted by $T_{p}$. Systems that are not able to ensure a pure delay $T_{p}$ lower than a tolerable upper bound $T_{\max }$ are considered to be unstable. The value of $T_{\max }$ can be estimated by tests in vehicles and simulations.

The behavioral reliability is estimated by the probability that the pure delay is greater than the maximum tolerable bound : $P_{B R}=P\left[T_{p}>T_{\max }\right]$. When $T_{p}$ is equal to or lower than $T_{\max }$, the quality of service is degraded while the vehicle is considered to be potentially unstable when $T_{p}>T_{\max }$.

\subsubsection{Evaluation of $T_{\max }$}

To illustrate the method, only the function "turning the wheels according to driver's request" is considered. The evaluation of $T_{\max }$ can be performed either by testing in a vehicle or by using Matlab/Simulink. The method we adopted was using Matlab/Simulink first, and confirming the results with testing in vehicles. The software framework used was composed of a Matlab/Simulink model of the Steer-by-Wire architecture and a vehicle environment model.

The architecture presented in section 4.3 was simulated according to a hand wheel angle utilization profile (positions of the hand wheel over time). The impact of the variation of $T_{p}$ on performance, mainly the stability of the vehicle and the time needed to reach the requested wheel angle, was evaluated and translated in terms of the "Quality of Service Score", denoted by $S$. Table 1 shows an example of the relation between the score $S$ given to the system and the perturbation time. It corresponds to an instantaneous rotation of the hand wheel from $0^{\circ}$ to $45^{\circ}$ at $100 \mathrm{~km} / \mathrm{h}$. From Table 1 , with a minimum tolerable score of 11 , one sees that $17.6 \mathrm{~ms}$ is the critical limit for this perturbation time: beyond this limit, the vehicle becomes unstable and the safety of the driver can be at risk. The different values of $T_{p}$ in Table 1 correspond to the cases where, during $1,2,4,5,6,7,8,9$ and 14 consecutive cluster cycles, the front axle actuators receive nothing (caused, for instance, by environmental perturbations). In practice, even without receiving any sampling data, the actuator still 
performs but the turning of the front axle is made, for instance, on the basis of the command of the previous period, or with an estimation based on the commands of several previous periods. So, in this case $T_{p}+T_{\text {mec }}$ is no longer the end-to-end delay strictly speaking, but the delay during which the system has not been able to take into account the current hand wheel angle in order to compute the commands for the actuators.

\begin{tabular}{|l|c|c|}
\hline $\begin{array}{c}\text { Configuration of } \\
\text { the Steering System }\end{array}$ & $T_{P}(\mathrm{~ms})$ & $\begin{array}{c}\text { Score } \\
S\end{array}$ \\
\hline $\begin{array}{l}\text { Mechanical steering } \\
\text { system }\end{array}$ & 0 & 11.23 \\
\hline Steer-by-Wire & 3.6 & 11.21 \\
\hline Steer-by-Wire & 5.6 & 11.19 \\
\hline Steer-by-Wire & 9.6 & 11.15 \\
\hline Steer-by-Wire & 11.6 & 11.13 \\
\hline Steer-by-Wire & 13.6 & 11.10 \\
\hline Steer-by-Wire & 15.6 & 11.05 \\
\hline Steer-by-Wire & $\mathbf{1 7 . 6}$ & $\mathbf{1 1}$ \\
\hline Steer-by-Wire & 19.6 & 10.90 \\
\hline Steer-by-Wire & 29.6 & 10.45 \\
\hline
\end{tabular}

Table 1: QoS score vs. perturbation time

\subsubsection{Quantification of the Behavioral Reliability}

With the use of the TTP/C network, communication cycles are predefined and cyclic. However, as will be shown by the analysis of the example shown in Figure, $T_{p}$ can be greater than a cluster cycle because of a possible desynchronization between the sampling period and the cluster cycle. The evaluation of the behavioral reliability should be based on the worst case $T_{p}$ but not the nominal $T_{p}$ because of the safety-critical nature of the system. Moreover, with transient failures due to perturbations, $T_{p}$ becomes a random variable. Therefore, in this section, we first evaluate $T p$ and then the behavioral reliability.

\section{Worst case pure delay without transient failures}


Figure shows the temporal characteristics of the front axle control function and the relationship with the cluster cycles. The Fault Tolerant Communication Layer (section 3.3.2) has been configured so that the front axle ECUs (FAA ECU1 and 2) have to wait for all replicas of data before consuming them.

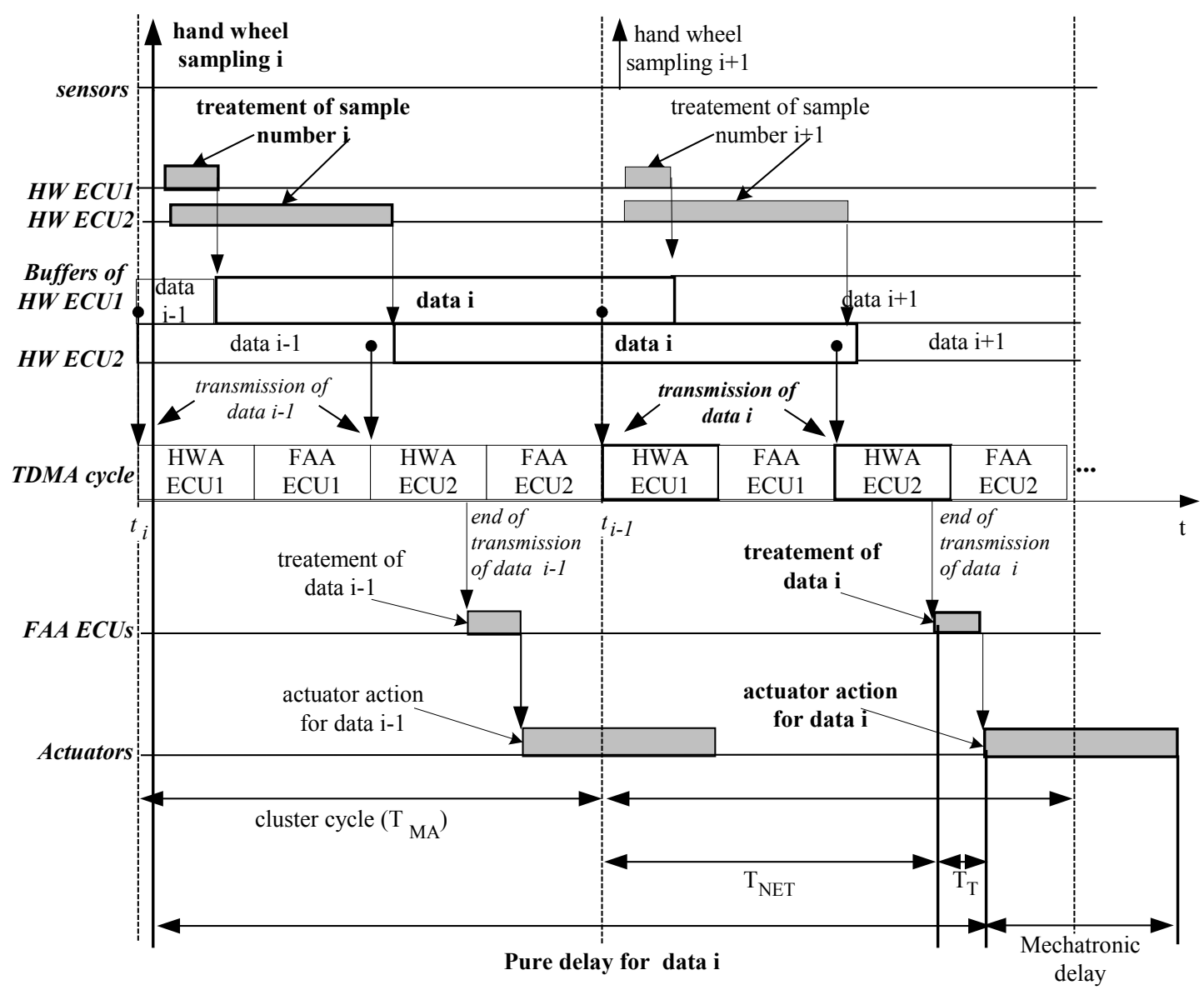

Figure 7: Temporal characteristics of the function "turning the wheels according to the driver's request"

Cluster cycles are numbered by index $i=1,2, \ldots$ The $i^{\text {th }}$ cluster cycle starts at $t_{i}$. For the front axle control function, the "worst case pure delay", denoted by $T_{p}^{W C}$, appears when the $i^{\text {th }}$ hand wheel sensor sampling period starts just after $t_{i}$. This slight desynchronization leads to a situation where, during the $i^{\text {th }}$ cluster cycle, only the data elaborated using the sample of the $(i-1)^{\text {th }}$ cluster cycle are transmitted to the front axle ECUs. In fact, in TTP/C, data kept in the buffer of each HW ECU is transmitted at the beginning of each slot. In Figure, at the beginning of each HWA ECU slot, data in the buffer has not 
yet been refreshed and only data corresponding to the previous sample is transmitted. So, the worst case delay between the $i^{\text {th }}$ HWA sample and the beginning of the $i^{\text {th }}$ actuation is given by:

$$
T_{p}^{W C}=T_{M A}+T_{N E T}+T_{T}
$$

where $T_{M A}$ is the duration of a cluster cycle, $T_{N E T}$ corresponds to the delay between the beginning of a cluster cycle and the arrival of all the replicas to the FAA ECU2, and where $T_{T}$ is the treatment time of the data within a FAA ECU.

Although, in our case, we have only one TDMA-round per cluster cycle, in general, one can find several TDMA-rounds per cluster cycle. In this latter case, assuming that there is one computation per data reception, the equation 1 takes the form :

$$
T_{p}^{W C}=n T_{r}+T_{N E T}+T_{T}
$$

where $T_{r}$ is the duration of a TDMA round and $n$ is the number of the TDMA-rounds between two HWA data emissions ( $n$ could be less than or equal to the number of TDMA-rounds in a cluster cycle). This result should be used for system dimensioning at the design step in order to ensure that $T_{p}{ }^{W C}$ is smaller than the tolerable upper bound $T_{\max }$ (e.g., $17.6 \mathrm{~ms}$ for the example presented in 4.4.5.1). For the slot placement given in Figure, we obtain:

$$
T_{W C P D}=1 * 2+1.4+0.2=3.6 \mathrm{~ms}
$$

This is to say that, in failure-free conditions, the pure delay is bounded by $3.6 \mathrm{~ms}$.

\section{Pure delay under transient failures and behavioral reliability evaluation}

When perturbations occur, the pure delay can be longer than $T_{p}^{W C}$, but how the perturbations will influence the pure delay depends on the failure occurrence model. Establishing a realistic failure model according to perturbation occurrences is a complex statistical work that is beyond the scope of this chapter. A more realistic failure model of EMI perturbations is proposed in [31].

In what follows, to illustrate the evaluation method of Behavioral Reliability $\left(P_{B R}\right)$, we use a simplified failure model, stationary in time and where failures are independent from each other. The granularity of the failure model is the Cluster Cycle (one failure leads to one erroneous or one empty cluster cycle). A failure can happen at any step detailed in Figure. Whichever step has a failure, we consider 
that no information is transmitted to the actuators during this cluster cycle (the information is lost or destroyed before the command is given to the actuators). In the worst case, each time a failure occurs, the actuator has to wait $T_{p}^{W C}$ plus one cluster cycle $T_{M A}$ to receive refreshed information. Let $T_{p}{ }^{W C, E R R}$ denotes the maximum delay with $N$ consecutive erroneous cluster cycles:

$$
T_{p}^{W C, E R R}=N T_{M A}+T_{p}^{W C}=N n T_{r}+T_{p}^{W C}
$$

Behavioral Reliability is then calculated by $P_{B R}=P\left[T_{p}{ }^{W C E R R}>\operatorname{Tmax}\right]=P\left[N\left(n T_{r}\right)+T_{p}{ }^{W C}>T_{\max }\right]$. So, we have:

$$
P_{B R}=P\left[N>\left\lfloor\left(T_{\max }-T_{p}^{W C}\right) / n T_{r}\right\rfloor\right]
$$

This probability can be directly used to determine the Safety Integrity Levels (SIL). In this case-study, the requirement is that $P_{B R}<10^{-9}$.

In our simplified failure model, as the failures occur following a stationary and uniform probability distribution, $P_{B R}$ also gives the probability of failures per hour. For the studied architecture, the maximum tolerable number of erroneous cluster cycles is given by:

$$
N=\left\lfloor\left(T_{\max }-T_{p}{ }^{W C}\right) /\left(n T_{r}\right)\right\rfloor=\lfloor(17.6-3.6) / 2\rfloor=7 .
$$

So, we must here have $P_{B R}=P[N>7]<10^{-9}$ (failure/hour).

As explained before, the chosen error model is a simplified one: erroneous or empty communication cycles are assumed independent events and the probability of losing one cluster cycle is $E_{R}$. So, the probability of losing $x$ consecutive cluster cycles before one successful transmission is $P[N=x]=(1$ $\left.-E_{R}\right)\left(E_{R}\right)^{x}$ (Geometric law)

$$
P_{B R}=P[N>x]=1-\sum_{k=0}^{x} P[N=k]=\left(E_{R}\right)^{x+1}
$$

The proposed operational architecture meets the dependability requirements with $E_{R}<0.075$ under a geometrical failure model. In practice, it is necessary to use a more realistic error model, constructed on the basis of measurements taken from a prototype. Indeed, the effects of transient failures and external perturbations, such as EMI or temperature peaks, are not negligible and will become even more problematic when the 42V technology [32] is used. 


\section{Conclusion}

X-by-Wire is a clear trend of automotive development due to the advantages of the electronic components for enhancing safety, functionality and reducing cost.

In this chapter, after having examined the real-time and dependability constraints of the X-by-Wire systems, we reviewed the fault-tolerant services and the communication protocols (TTP/C, FlexRay and TTCAN) that are needed for such systems. Methods for designing a dependable X-by-Wire system were described and a Steer-by-Wire system based on TTP/C was then used as a case study. We showed how to build a fault-tolerant architecture by choosing the necessary redundant components and the schedule of transmission. A method for evaluating the probability that the real-time constraints would be violated under a simple perturbation model was also proposed. This method can be used to predict whether the architecture meets the SIL4 requirement.

If the dependability of the X-by-Wire can be evaluated by assuming that one can establish a realistic failure model, the certification organization still remains to be convinced. At the time of writing, the legislation in some countries does not authorize fully X-by-Wire cars to circulate. The use of X-byWire systems mass production cars in the future also depends on other factors such as the advances in the $42 \mathrm{~V}$ technology.

\section{References}

1 Society of Automotive Engineers (SAE) public discussion: 42 Volt Electrical Systems et Fuel Cells : Harmonious marriage or incompatible partners ?, SAE (N.Traub), General Motors (Ch.Borroni-Bird, Director of Design and Technology Fusion), Delphi (J.Botti, Innovation Center), Daimler Chrysler (T.Moore, Vice President, Liberty and Technical Affairs), UTC Fuels Cells (F.R.Preli, Vice President, Engineering), SAE 2003 World Congress \& Exhibition, Detroit (USA), 2003.

2 IEC61508-1, Functional Safety of Electrical Electronic Programmable Electronic Safety-related Systems Part 1 : General requirements, IEC/SC65A, 1998.

3 J. Rushby, A Comparison of Bus Architectures for Safety-Critical Embedded Systems, Technical report, Computer Science Laboratory SRI International, 2003. 
4 E. Dilger, T. Führer, B. Müller, S. Poledna, The X-By-Wire Concept: Time-Triggered Information Exchange and Fail Silence Support by new System Services, Technische Universität Wien, Institut für Technische Informatik, n7/1998, also available as SAE Technical Paper 98055, 1998.

5 C. Temple, Avoiding the Babbling-Idiot Failure in a Time-Triggered Communication System, International Symposium on Fault-Tolerant Computing (FTCS), Munich (Germany), 1998.

6 S. Poledna, P. Barrett, A. Burns, A. Wellings, Replica Determinism and Flexible Scheduling in Hard RealTime Dependable Systems, IEEE Transactions on Computers, vol.49, n², pp100-111, 2000.

7 Time-Triggered Protocol TTP/C, High-Level Specification Document, Protocol Version 1.1, 2003.

8 H. Kopetz, Real-Time Sytems: Design Principles for Distributed Embedded Applications, Kluwer Academic Publishers, 1997.

9 H. Pfeifer, Formal Verification of the TTP Group Membership Algorithm, FORTE/PSTV Euroconference, Pisa (Italy), 2000.

10 H. Kopetz, R. Nossal, R. Hexel, A Krüger, D. Millinger, R.Pallierer, C. Temple, M. Krug, Mode Handling in the Time-Triggered Architecture, Control Engineering Practice, vol.6, pp61-66, 1998.

11 TTTech Computertechnik AG, http://www.tttech.com/, 2004.

12 G. Bauer, M. Paulitsch, An Investigation of Membership and Clique Avoidance in TTP/C, 19th IEEE Symposium on Reliable Distributed Systems, Nuremberg (Germany), 2000.

13 FlexRay Consortium, http://www.flexray.com, 2004.

14 Road vehicles - Controller area network (CAN) - Part 4 : Time Triggered Communication - ISO 11898-4.

15 Bosch - Time Triggered Communication on CAN, http://www.can.bosch.com/content/TT_CAN.html, 2004.

16 OSEK Consortium, OSEK/VDX Time-Triggered Operating System, Version 1.0, available at http://www.osek-vdx.org/, 2001.

17 OSEK Consortium, OSEK/VDX Fault-Tolerant Communication, Version 1.0, available at http://www.osekvdx.org/, 2001.

18 N. Tracey, Comparing OSEK and OSEKTime, Embedded System Conference (ESC) Europe, Stuttgart, 2001.

19 X-by-Wire Project, Brite-EuRam 111 Program, X-By-Wire - Safety Related Fault Tolerant Systems in Vehicles, Final Report, 1998. 
20 A. Avizienis, J.-C. Laprie and B. Randell, "Fundamental Concepts of Dependability", in 3rd Information Survivability Workshop, (Boston, MA, USA), pp 7-12, IEEE Computer Society Press, 2000.

21 J. A. Garay, K. J. Perry, A Continuum of Failure Models for Distributed Computing, 6th Distributed Algorithm International Workshop (WDAG), Haifa (Israel), 1992.

22 L. Lamport, R. Shostak, M. Pease, The Byzantine Generals Problem, ACM Transactions on Programming Language and Systems, vol. 4, no. 3, pp382-401, 1982.

23 G. Grünsteidl, H. Kantz, H. Kopetz, Communication Reliability in Distributed Real-Time systems, 10th IFAC Workshop on Distributed Computer Control Systems, Semmering (Austria), 1991.

24 P. Herout,S, Racek, J. Hlavicka, Model-based Dependability Evaluation Method for TTP/C Based Systems, EDCC-4 - Fourth European Dependable Computing Conference, Toulouse (France), 2002.

25 R. Hexel, FITS: a Fault Injection Architecture for Time-Triggered Systems, 26th Australian Computer Science Conference (ACSC2003), Adelaide (Australia), 2003.

26 Report by the Inquiry Board, Ariane 501 Flight Failure, http://www.mssl.ucl.ac.uk/www_plasma/missions/cluster/about_cluster/cluster1/ariane5rep.html, 1996.

27 B. Gaujal, N. Navet, Maximizing the Robustness of TDMA Networks with Application to TTP/C, Technical Report RR-4614, INRIA, 2002.

28 B. Gaujal, N. Navet, Optimal Replica Allocation for TTP/C BasedSystems, 5th FeT IFAC Conference (FeT'2003), Aveiro (Portugal), July 2003.

29 R. Hammett, P. Babcock, Achieving 10-9 Dependability With Drive-By-Wire Systems, SAE 2003 World Congress \& Exhibition, Detroit (USA), 2003.

30 C. Wilwert, Y.Q. Song, F. Simonot-Lion, T. Clément, Evaluating Quality of Service and Behavioral Reliability of Steer-by-Wire systems, 9th IEEE International Conference on Emerging Technologies and Factory Automation (ETFA), Lisbon (Portugal), 2003.

31 N. Navet, Y.Q. Song, F. Simonot, Worst-case Deadline Failure Probability in Real-Time Applications Distributed over CAN (Controller Area Network), Journal of Systems Architecture, vol. 46, pp607-617, 2000.

32 H. Kopetz, H. Kantz, G. Gründsteidl, P. Puschner, J. Reisinger, Tolerating Transient Fault in MARS, 20th Symposium of Fault Tolerant Computing, Newcaste upon Tyne, UK, 1990. 